\title{
Respostas de tipos funcionais de plantas à intensidade de pastejo em vegetação campestre
}

\author{
Enio Egon Sosinski Júnior ${ }^{(1)}$ e Valério DePatta Pillar ${ }^{(1)}$
}

\begin{abstract}
(1)UFRGS, Instituto de Biociências, Dep. de Ecologia, Av. Bento Gonçalves, 9500, CEP 91540-000 Porto Alegre, RS. E-mail: esosinski@ecologia.ufrgs.br, vpillar@ecologia.ufrgs.br
\end{abstract}

\begin{abstract}
Resumo - O objetivo deste trabalho foi identificar respostas da vegetação campestre, caracterizada por espécies e tipos funcionais de planta, a diferentes intensidades de pastejo. O levantamento da vegetação, usando quadros de $0,25 \mathrm{~m}^{2}$, foi realizado na primavera e no verão. A descrição da estrutura da vegetação envolveu a identificação das espécies presentes em cada quadro, a estimativa de sua abundância-cobertura e a descrição das espécies considerando 21 atributos macromorfológicos qualitativos e quantitativos. A análise dos dados objetivou encontrar um subconjunto ótimo de atributos, definindo os tipos funcionais de forma a maximizar a congruência $\rho(\mathrm{D} ; \Delta)$ entre a variação da vegetação (matriz $\mathrm{D}$ ) e do fator oferta de forragem (matriz $\Delta$ ). A análise de ordenação permitiu a identificação de tipos funcionais que apresentaram respostas mais evidentes, em termos de abundância-cobertura. A composição da vegetação, descrita pelos tipos funcionais, foi comparada entre níveis de oferta de forragem, por análise de variância multivariada com testes de aleatorização, sendo detectadas diferenças significativas $(P=0,002)$. Quando a composição da vegetação foi descrita por espécies não foram observadas diferenças significativas. A utilização de tipos funcionais permite detectar efeito da intensidade de pastejo, não evidenciado em uma análise baseada na composição por espécies.
\end{abstract}

Termos para indexação: meio ambiente, atributo morfológico, forragem, manejo de pastagem.

\section{Response of plant functional types to grazing intensity in grassland}

\begin{abstract}
The objective of this work was to identify responses of grassland vegetation, described by species and plant functional types, to different grazing intensities. In order to describe the vegetation, $0.25 \mathrm{~m}^{2}$ quadrats were used in the spring and summer. Vegetation description involved identification and cover-abundance estimation of the species in each quadrat. Each species was described by 21 macro-morphological qualitative and quantitative attributes. Data analysis aimed at identifying functional types as a subset of attributes that maximized the congruence $\rho(\mathrm{D} ; \Delta)$, between the variation of the vegetation (matrix $\mathrm{D}$ ) and of the forage available (matrix $\Delta$ ). Ordination revealed plant functional types that, concerning cover-abundance, responded more clearly to this factor. Multivariate analysis of variance with randomization testing was used to compare the vegetation composition, as described by plant functional types, among the forage available levels, and significant differences $(P=0.002)$ were observed. The differences were not significant when vegetation was described by the species composition. The use of plant functional types is more effective to detect the result of grazing intensity than the use of species composition.
\end{abstract}

Index terms: environment, morphological traits, forage, grassland management.

\section{Introdução}

A descrição de comunidades vegetais, relacionando formas com o ambiente baseia-se na observação de que fatores físicos e biológicos do meio são determinantes da fisionomia da vegetação. $\mathrm{O}$ ambiente funciona como um filtro, removendo os indivíduos que não apresentam determinada adaptação (Keddy, 1992). A presença e abundância dos indivíduos podem ser interpretadas como uma resposta à variação dos fatores (Díaz et al., 1992; Pillar, 1999).

Com o objetivo de desenvolver modelos globais de predição de mudanças na vegetação frente às mudanças climáticas, estudos recentes têm procurado identificar padrões e conectar os diversos biomas do globo, baseando-se na suposição de que a evolução das comunidades vegetais seria convergente. O International Geosphere-Biosphere Programme (IGBP), com seu 
núcleo de estudos principal Global Change and Terrestrial Ecosystems (GCTE), tem organizado grupos de trabalhos, desde 1993, sobre o assunto, chegando à conclusão de que a identificação de tipos funcionais (TF) de plantas é essencial para os modelos preditivos (Steffen et al., 1992). Vários estudos têm demonstrado que dificilmente será possível estabelecer uma classificação universal de TF e, portanto, eles devem ser identificados para diferentes propósitos e com diferentes níveis de resolução (Skarpe, 1996). Segundo alguns autores, TF são grupos de plantas que exibem respostas similares às condições de ambiente e apresentam efeitos parecidos sobre os processos dominantes do ecossistema. Tipos funcionais de planta são os funcionalmente similares (Box, 1996), ou seja, aqueles que permitem a percepção mais nítida da associação entre vegetação e ambiente (Pillar, 1999).

Pesquisas recentes têm quantificado o caráter adaptativo da vegetação por meio do uso de atributos morfológicos. Foram desenvolvidos métodos de descrição da vegetação com TF de plantas como alternativa ou complemento com espécies (Pillar \& Orlóci, 1993). Por meio de modelos matemáticos, são avaliadas as diferentes estratégias adaptativas sob diferentes limitações ambientais. A forma analítica de selecionar os atributos (Pillar, 1999; Pillar \& Orlóci,1993) é baseada na maximização da congruência entre a variação da vegetação descrita por TF e a variação ambiental. Partindose da matriz de s espécies por $\mathrm{k}$ atributos, pré-selecionados, uma matriz de abundância de s populações (que podem ser espécies) por $\mathrm{n}$ unidades amostrais e outra de $\mathrm{p}$ variáveis ambientais por $\mathrm{n}$ unidades amostrais, com o uso de um algoritmo recursivo, pode-se determinar a sequiência ótima dos atributos e sua relevância ecológica. Baseado neste esquema analítico quantitativo, é possível derivar um subconjunto ótimo de atributos, definindo tipos funcionais.

Qualquer que seja a aproximação escolhida para a definição dos tipos de planta, é vital a seleção do conjunto de atributos que será submetido ao esquema analítico (Skarpe, 1996). A escolha do conjunto de atributos pode estar baseada em dados da literatura ou em estudos de campo (Díaz Barradas et al., 1999) ou laboratório (Grime et al., 1997). Podem ser utilizados atributos morfológicos ou fisiológicos ligados às características ecológicas (Keddy, 1992), porém deve-se levar em conta os custos e a possibilidade da observação (Skarpe, 1996). Dependendo da escala e propósito do estudo, podem ser coletados atributos de cada espécie ou, consideran- do a variação intra-específica, de cada população (Thompson et al., 1996; Díaz et al., 1999).

Quando se estuda TF em resposta à intensidade de pastejo, sendo esta uma função inversa da oferta de forragem, procura-se uma combinação de atributos que permitam à planta persistir sob uma determinada intensidade que configure distúrbio. Diferentes combinações de atributos morfológicos afetam a escolha destas plantas pelos animais, influindo na probabilidade da espécie ser pastejada. Com isso, alteram-se as abundâncias relativas e os estágios fenológicos (Boggiano, 1995).

Nas plantas, o dano pode ser evitado por meio de estratégias de controle e de tolerância. A primeira é alcançada por mecanismos de defesa e escape, como atributos relacionados à arquitetura, compostos bioquímicos, ou associações de plantas. Tais mecanismos reduzem a probabilidade do acesso aos tecidos da planta e a palatabilidade do material aos animais. Relacionados à defesa, encontram-se os atributos que restringem a ação do animal sobre a planta, como taninos, alcalóides ou óleos secundários, ou ainda atributos anatômicos e estruturais como pilosidade, espinhos, cerosidade, esclerofilia, e silificação. Por sua vez, a associação interespecífica permite proteção a espécies com menor expressão dos mecanismos de defesa, quando crescem próximas de espécies com grande expressão destes mecanismos. Por estratégias de tolerância tem-se a capacidade de rebrote das plantas após o dano (Briske \& Richards, 1995; McIntyre et al., 1999b).

O objetivo deste trabalho foi identificar respostas a diferentes intensidades de pastejo na vegetação campestre, descrita por espécies e TF de plantas.

\section{Material e Métodos}

$O$ levantamento foi realizado em um experimento de pastejo, na Estação Experimental Agronômica (EEA) da Universidade Federal do Rio Grande do Sul, no Município de Eldorado do Sul, RS, situada na região fisiográfica denominada Depressão Central. Os solos da área experimental são descritos como solos rasos de textura franco-arenosa, imperfeitamente drenados e presentes em relevos suavemente ondulados que, segundo a classificação brasileira de solos, podem ser definidos como Plintossolo (Embrapa, 1999). Por ocasião da implantação do experimento, em maio de 1996, foi feita a correção da acidez do solo e adubação de base $(\mathrm{N}, \mathrm{P}, \mathrm{K})$, conforme a recomendação para forrageiras de verão. A vegetação da área do experimento pode 
ser considerada como um campo nativo regenerado, pois as espécies predominantes mostram muita similaridade com este tipo de vegetação (Boggiano, 2000).

Os níveis do fator oferta de forragem (OF) utilizados foram: 4,0, 5,5, 9,0, 12,5 e 14,0 kg de matéria seca verde (MSV) por $100 \mathrm{~kg}$ de peso vivo (PV) por dia, em um delineamento central composto, com o tratamento central $(9,0 \%$ PV de OF) sendo repetido seis vezes e os demais sendo repetidos duas vezes. Os tratamentos foram conduzidos em 14 potreiros com a área estimada de tal maneira que fosse possível manter, pelo menos, $200 \mathrm{~kg}$ de PV por dia, durante três dias.

As unidades amostrais, dentro de cada potreiro, foram quadros de $0,25 \mathrm{~m}^{2}(0,5 \mathrm{x} 0,5 \mathrm{~m})$. Os quadros foram locados em cada um dos 14 potreiros avaliados, sistematicamente, ao longo de um eixo central longitudinal estabelecido de forma aproximada. Os quadros foram marcados de forma a manter uma distância mínima de $4 \mathrm{~m}$ da borda da parcela. Foram marcados cinco quadros em cada um dos potreiros, totalizando 70 quadros.

O levantamento da vegetação foi realizado na primavera de 1999, de 13 a 21 de novembro, quando foram descritos três quadros em cada potreiro e no verão de 2000, de 6 a 9 de janeiro, quando foram descritos mais dois quadros em cada potreiro. Os levantamentos foram realizados quando os potreiros estavam sem a presença do gado bovino por 20 dias, no terço final do período de descanso da pastagem. Os quadros adicionais amostrados, no verão, foram intercalados aos quadros da primavera.

A descrição da estrutura da vegetação foi feita com base no levantamento de espécies de vegetais vasculares. Todas as espécies presentes em cada quadro foram anotadas, procurando-se identificá-las até o nível de espécie. As espécies que não foram localmente identificadas, foram coletadas fora dos quadros e herborizadas para posterior identificação, com auxílio de bibliografia e consultas a especialistas. A abundânciacobertura de cada espécie foi estimada visualmente por meio da escala de Braun-Blanquet (1964).

Cada espécie presente no quadro foi caracterizada por seus atributos macromorfológicos qualitativos e quantitativos (Tabela 1). Quando não foi possível ou conveniente descrever a campo o estado de atributos pouco variáveis dentro de espécies, procurou-se orientação na literatura. Nesse caso, o estado do atributo foi considerado idêntico para todos os indivíduos da mesma espécie. Todos os atributos, mesmo quantitativos, foram categorizados em um número não muito elevado de classes, para permitir a análise dos dados, conforme Pillar \& Orlóci (1993).

$\mathrm{Na}$ análise dos dados de abundância-cobertura, as classes da escala de Braun-Blanquet (1964) foram transformadas para a escala Maarel (1979), a qual atribui valores $1,2,3,5,7,8$ ou 9 , respectivamente, para $\mathrm{r},+, 1$, 2, 3, 4 ou 5 da outra escala.

O algoritmo de otimização (Pillar \& Orlóci, 1993; Pillar, 1999) foi utilizado na obtenção do conjunto ótimo de atributos. Por meio da sub-rotina "ranking", implementada no aplicativo SYNCSA (Pillar, 2000), determinou-se o subconjunto de atributos que maximizou a congruência $\rho(D ; \Delta)$ entre a variação da vegetação e o fator oferta de forragem. O valor de congruência é o coeficiente de correlação de Pearson calculado entre as matrizes D e $\Delta$, semelhante à estatística padronizada de Mantel (1967). A matriz D contém as dissimilaridades (distância Euclidiana) entre as comunidades descritas pelos TF. A matriz $\Delta$ contém as dissimilaridades (diferenças em valores absolutos) entre os níveis de oferta de forragem (Pillar, 1999). Com base na identificação da ordem ótima do conjunto de atributos, foi determinado o subconjunto ótimo de atributos que melhor define os TF (Pillar, 1999) para esse fator.

A análise exploratória multivariada foi usada para identificar as principais tendências de variação descrita pelos TF definidos pelo melhor conjunto de atributos para o fator oferta de forragem. Para isso, a matriz com a performance média dos TF ótimos em cada potreiro foi submetida à análise de ordenação pelo método de análise de coordenadas principais (PCoA), usando o aplicativo SYNCSA. Para fins de comparação as análises foram também feitas com a composição de espécies.

A análise de variância multivariada com teste de aleatorização foi aplicada aos dados para testar a hipótese de independência da composição dos potreiros em relação ao fator oferta de forragem. Foram testadas as composições baseadas nos TF e nas espécies. Optouse pelo uso do teste de aleatorização por este evitar a pressuposição de que os dados apresentam uma distribuição das probabilidades teóricas baseados na Normal (Pillar \& Orlóci, 1996).

\section{Resultados e Discussão}

No levantamento dos 70 quadros, foram descritas 827 populações diferentes quanto aos atributos avalia- 
dos, sendo 547 populações quando foram amostrados os 42 quadros na primavera (novembro de 1999) e 280 populações quando foram amostrados os demais 28 quadros no verão (janeiro de 2000). Foram identificadas 81 espécies em 24 famílias, predominando a família Poaceae, com 23 espécies, e $28 \%$ da riqueza total, seguida pela família Asteraceae com 14 espécies e $17 \%$ da riqueza total e Fabaceae com oito espécies e $10 \%$ da riqueza total (Tabela 2).

Na Figura 1 são apresentados os perfis da função que avalia a congruência, conforme o nível de agregação das unidades amostrais e a época de amostragem. Cada perfil apresenta a ordem ótima do conjunto de atributos (da direita para esquerda) e os valores de congruência, considerando os atributos no processo cumulativo. Os atributos que determinam congruência máxima definem o subconjunto ótimo de atributos para uma determinada forma de análise. Foram gerados dois perfis para cada época de amostragem (Figura 1: a, d- duas épocas conjuntamente; $\mathrm{b}, \mathrm{e}-$ novembro; $\mathrm{c}, \mathrm{f}-\mathrm{ja}-$ neiro). Na Figura 1(a, b, c) observam-se os perfis, considerando os quadros individualmente, e na Figura 1(d, e, f), com análise da média dos quadros em cada potreiro. Os valores de congruência máxima obtida com os quadros individualmente foram mais baixos, independentemente da época de amostragem. As análises com a média dos quadros equivalem a um aumento do tamanho do quadro. Esse efeito de escala ficou evidenciado nos resultados e, provavelmente, está relacionado a uma redução do nível de indeterminação (Pillar \& Orlóci, 1991) e de não-linearidade (Pillar, 1999).

Os valores de congruência para um mesmo nível de agregação são similares quando comparados entre si na Figura 1(a, b, c). Ou seja, observa-se maior efeito, sobre o valor de congruência, do nível de agregação dos quadros do que da época de levantamento. A inclusão do levantamento de janeiro não modificou as tendências que haviam sido encontradas no levantamento de no-

Tabela 1. Atributos utilizados na descrição da estrutura da vegetação sob efeito da oferta de forragem.

\begin{tabular}{|c|c|c|c|c|}
\hline $\begin{array}{l}\text { Forma } \\
\text { geral }\end{array}$ & Atributos & Código & Estados dos atributos & $\begin{array}{c}\text { Fonte } \\
\text { dos dados }\end{array}$ \\
\hline \multirow[t]{4}{*}{$\begin{array}{l}\text { Forma } \\
\text { de vida }\end{array}$} & Posição das gemas & $\mathrm{pg}$ & $\begin{array}{l}\text { 1:Fanerófita; 2:Caméfita; 3:Hemicriptófita; } \\
\text { 4:Geófita; 5:Terófita }\end{array}$ & (Burkart, 1969) \\
\hline & Persistência & pe & $0:$ Não perene; $1:$ Perene & (Burkart, 1969) \\
\hline & Órgãos de reserva & or & 0:Ausentes; 1:Presentes & (Burkart, 1969) \\
\hline & Sazonalidade & Sz & 0:Hibernal; 1:Estival & (Burkart, 1969) \\
\hline \multirow{5}{*}{$\begin{array}{l}\text { Forma } \\
\text { da planta }\end{array}$} & Forma de crescimento & fc & 1:Solitária; 2:Rosulada; 3:Cespitosa & Campo \\
\hline & Inclinação do caule & ip & 1:Prostrado; 2:Ereto; 3:Semi-ereto & Campo \\
\hline & Propagação vegetativa & $\mathrm{pv}$ & 1:Estoloníferas; 2:Rizomatosas; 3:Outras & Campo \\
\hline & Altura da biomassa & $\mathrm{ab}$ & $\begin{array}{l}1:<2,0 ; 2: 2,0<3,5 ; 3: 3,5<6,5 ; 4: 6,5<12,0 ; 5: 12,0<22,5 \\
6:>22,5 \mathrm{~cm}\end{array}$ & Campo \\
\hline & Comprimento da bainha & $\mathrm{cb}$ & $1:<0,7 ; 2: 0,7<1,5 ; 3: 1,5<3,0 ; 4: 3,0<5,4 ; 5: 5,4<9,5 ; 6:>9,5 \mathrm{~cm}$ & Campo \\
\hline \multirow{5}{*}{$\begin{array}{l}\text { Forma } \\
\text { da lâmina } \\
\text { foliar }\end{array}$} & Secção transversal da lâmina & st & 1:Plana; 2:Dobrada; 3:Enrolada; 4:Outras (afilas) & Campo \\
\hline & Comprimento da lâmina & $\mathrm{cl}$ & $1:<0,8 ; 2: 0,8<2,0 ; 3: 2,0<4,2 ; 4: 4,2<7,9 ; 5: 7,9<14,4 ; 6:>14,4 \mathrm{~cm}$ & Campo \\
\hline & Largura da lâmina & 11 & $1:<0,4 ; 2: 0,4<0,8 ; 3: 0,8<1,5 ; 4: 1,5<2,4 ; 5: 2,4<3,5 ; 6:>3,5 \mathrm{~cm}$ & Campo \\
\hline & Área da lâmina & al & $\begin{array}{l}1:<1,0 ; 2: 1,0<3,1 ; 3: 3,1<7,3 ; 4: 7,3<15,7 ; 5: 15,7<32,8 \\
6:>32,8 \mathrm{~cm}\end{array}$ & Calculado \\
\hline & Formato da lâmina & fl & $\begin{array}{l}1:<0,22 ; 2: 0,22<0,49 ; 3: 0,49<0,81 ; 4: 0,81<1,31 ; 5: 1,31<1,68 ; \\
6:>1,68 \mathrm{~cm}\end{array}$ & Calculado \\
\hline \multirow{7}{*}{$\begin{array}{l}\text { Lignificação } \\
\text { e defesa } \\
\text { da planta }\end{array}$} & Tipo de tecido dos caules aéreos & tc & 1:Herbáceo; 2:Lenhoso; 3:Sem caule & Campo \\
\hline & Textura da lâmina & tl & $\begin{array}{l}\text { 1:Membranácea; 2:Herbácea; 3:Coriácea; 4:Suculenta; 5:Fibrosa; } \\
\text { 6:Outras (afilas) }\end{array}$ & Campo \\
\hline & Superfície dorsal da lâmina & sd & 1:Lisa; 2:Rugosa; 3:Pubescente; 4:Tomentosa; 5:Afila & Campo \\
\hline & Superfície ventral da lâmina & sv & 1:Lisa; 2:Rugosa; 3:Pubescente; 4:Tomentosa; 5:Afila & Campo \\
\hline & Indumento da lâmina & in & 1:Áspero; 2:Espinhoso; 3:Outros & Campo \\
\hline & Resistência da lâmina à tensão & $\mathrm{rl}$ & $\begin{array}{l}1:<0,25 ; 2: 0,25<0,75 ; 3: 0,75<1,5 ; 4: 1,5<2,25 ; 5: 2,25<3,75 \\
6:>3,75\end{array}$ & Campo \\
\hline & Tensão da lâmina (estimado) & ts & 1 a 4 & Campo \\
\hline
\end{tabular}


Tabela 2. Tipos funcionais (TF) determinados pelas análises, identificando as espécies que os compõem, as classes dos atributos, resistência da lâmina à tensão $(\mathrm{RL})$ e persistência (PE), que definem o subconjunto ótimo e o respectivo grupo ecológico (GE).

\begin{tabular}{|c|c|c|c|c|}
\hline T F & GE & $\mathrm{RL}^{(1)}$ & $\mathrm{PE}^{(2)}$ & Família e espécie \\
\hline 1 & - & 3 & 1 & $\begin{array}{l}\text { Amaranthaceae: Pfaffia tuberosa; Asteraceae: Aspilia montevidensis, Elephantopus mollis, Hypochoeris sp., } \\
\text { Orthopappuss angustifolius, Pterocaulon sp., Vernonia nudiflora; Convolvulaceae: Dichondra sericea; } \\
\text { Cyperaceae: Cyperus brevifolius; Fabaceae: Clitoria nana, Crotalaria tweediana, Desmodium incanum, } \\
\text { Macroptilium prostratum; Hipoxidaceae: Hypoxis decumbens; Iridaceae: Herbertia pulchella, } \\
\text { Sisyrinchium sp.; Plantaginaceae: Plantago tomentosa; Poaceae: Aristida laevis, Axonopus affinis, Briza } \\
\text { subaristata, Chloris sesquiflora, Coelorhachis selloana, Cynodon dactylon, Eleusine thristachya, Paspalum } \\
\text { dilatatum, Paspalum notatum, Paspalum pauciciliatum, Paspalum saurae, Paspalum plicatulum, Paspalum } \\
\text { paniculatum, Paspalum urvillei, Schizachyrium microstachyum, Sporobolus indicus; Umbelliferae: } \\
\text { Eryngium sanguisorba. }\end{array}$ \\
\hline 2 & 1 & 2 & 1 & $\begin{array}{l}\text { Acanthaceae: Ruellia sp.; Amaranthaceae: Pfaffia tuberosa; Asteraceae: Aspilia montevidensis, } \\
\text { Elephantopus mollis, Hypochoeris sp. Orthopappuss angustifolius, Pterocaulon sp., Vernonia nudiflora; } \\
\text { Convolvulaceae: Dichondra sericea; Cyperaceae: Cyperus brevifolius; Fabaceae: Crotalaria tweediana, } \\
\text { Desmodium incanum, Macroptilium prostratum, Stylosanthes montevidensis; Hipoxidaceae: Hypoxis } \\
\text { decumbens; Iridaceae: Cypella sp., Herbertia pulchella, Sisyrinchium sp.; Labiatae: Scutellaria racemosa; } \\
\text { Malvaceae: Sida rhombifolia; Melastomataceae: Tibouchina gracilis; Plantaginaceae: Plantago tomentosa; } \\
\text { Poaceae: Axonopus affinis, Briza subaristata, Chloris sesquiflora, Coelorhachis selloana, Cynodon dactylon, } \\
\text { Eleusine thristachya, Panicum hians, Paspalum notatum, Paspalum plicatulum, Paspalum paniculatum, } \\
\text { Piptochaetium montevidense, Setaria geniculata, Sporobolus indicus; Rubiaceae: Richardia humistrata; } \\
\text { Sterculiaceae: Waltheria douradinha. }\end{array}$ \\
\hline 3 & 2 & 5 & 1 & $\begin{array}{l}\text { Asteraceae: Elephantopus mollis, Hypochoeris sp., Orthopappuss angustifolius; Fabaceae: Desmodium } \\
\text { incanum; Iridaceae: Herbertia pulchella; Poaceae: Andropogon lateralis, Axonopus affinis, Paspalum } \\
\text { notatum, Paspalum pauciciliatum, Paspalum plicatulum, Paspalum paniculatum, Paspalum urvillei; } \\
\text { Umbelliferae: Eryngium horridum, Eryngium sanguisorba. }\end{array}$ \\
\hline 4 & 1 & 1 & 1 & $\begin{array}{l}\text { Acanthaceae: Ruellia sp.; Amaranthaceae: Pfaffia tuberosa; Asteraceae: Aspilia montevidensis, Baccharis } \\
\text { cf. dracunculifolia, Elephantopus mollis, Hypochoeris sp., Orthopappuss angustifolius, Pterocaulon sp., } \\
\text { Solidago chilensis, Vernonia nudiflora; Convolvulaceae: Dichondra sericea, Evolvulus sericeus; } \\
\text { Cyperaceae: Cyperus brevifolius, Cyperus cayennensis; Fabaceae: Crotalaria tweediana, Desmanthus } \\
\text { depressus, Desmodium incanum; Hipoxidaceae: Hypoxis decumbens; Iridaceae: Cypella sp., Herbertia } \\
\text { pulchella, Sisyrinchium sp.; Juncaceae: Juncus sp.; Labiatae: Scutellaria racemosa; Lythraceae: Cuphea } \\
\text { cf. glutinosa; Malvaceae: Sida rhombifolia; Oxalidaceae: Oxalis eriocarpa; Plantaginaceae: Plantago } \\
\text { tomentosa; Poaceae: Aristida laevis, Axonopus affinis, Briza subaristata, Coelorhachis selloana, Cynodon } \\
\text { dactylon, Paspalum plicatulum, Panicum sabulorum, Piptochaetium montevidense, Setaria geniculata, Setaria } \\
\text { vaginata; Polygonaceae: Rumex obtusifolius; Rubiaceae: Borreria eryngioides, Borreria laxa, Borreria } \\
\text { verticillata, Galianthe fastigiata, Relbunium richardianum, Richardia humistrata; Umbelliferae: Centella } \\
\text { asiatica; Verbenaceae: Verbena cf. montevidensis, Verbena sp. }\end{array}$ \\
\hline 5 & 2 & 4 & 1 & $\begin{array}{l}\text { Asteraceae: Baccharis trimera, Elephantopus mollis, Hypochoeris sp., Orthopappuss angustifolius; } \\
\text { Convolvulaceae: Dichondra sericea; Cyperaceae: Cyperus brevifolius, Cyperus cayennensis, Cyperus } \\
\text { sesquiflorus; Fabaceae: Desmodium incanum, Macroptilium prostratum; Iridaceae: Sisyrinchium sp.; } \\
\text { Plantaginaceae: Plantago tomentosa; Poaceae: Andropogon lateralis, Aristida laevis, Axonopus affinis, } \\
\text { Chloris sesquiflora, Coelorhachis selloana, Paspalum notatum, Paspalum pauciciliatum, Paspalum } \\
\text { plicatulum, Paspalum paniculatum, Paspalum urvillei, Schizachyrium microstachyum; Umbelliferae: } \\
\text { Eryngium horridum, Eryngium sanguisorba. }\end{array}$ \\
\hline 6 & 1 & 1 & 0 & $\begin{array}{l}\text { Asteraceae: Conyza sp., Facelis retusa, Gamochaeta sp., Gnaphalium spicatum, Soliva pterosperma; } \\
\text { Caryophyllaceae: Spergula arvensis; Convolvulaceae: Dichondra sericea; Fabaceae: Lupinus linearis, } \\
\text { Trifolium dubium; Poaceae: Lolium multiflorum; Primulaceae: Anagallis arvensis; Scrophulariaceae: } \\
\text { Linaria sp.; Umbelliferae: Apium leptophyllum. }\end{array}$ \\
\hline 7 & 2 & 6 & 1 & Poaceae: Paspalum urvillei; Umbelliferae: Eryngium horridum. \\
\hline 8 & - & 2 & 0 & eae: Drymaria cordata; Poaceae: Lolium multiflorum. \\
\hline
\end{tabular}

(1) $1:<0,25 ; 2: 0,25-0,75 ; 3: 0,75-1,5 ; 4: 1,5-2,25 ; 5: 2,25-3,75 ; 6:>3,75$. ${ }^{(2)} 0$ : não perene; 1 : perene. 

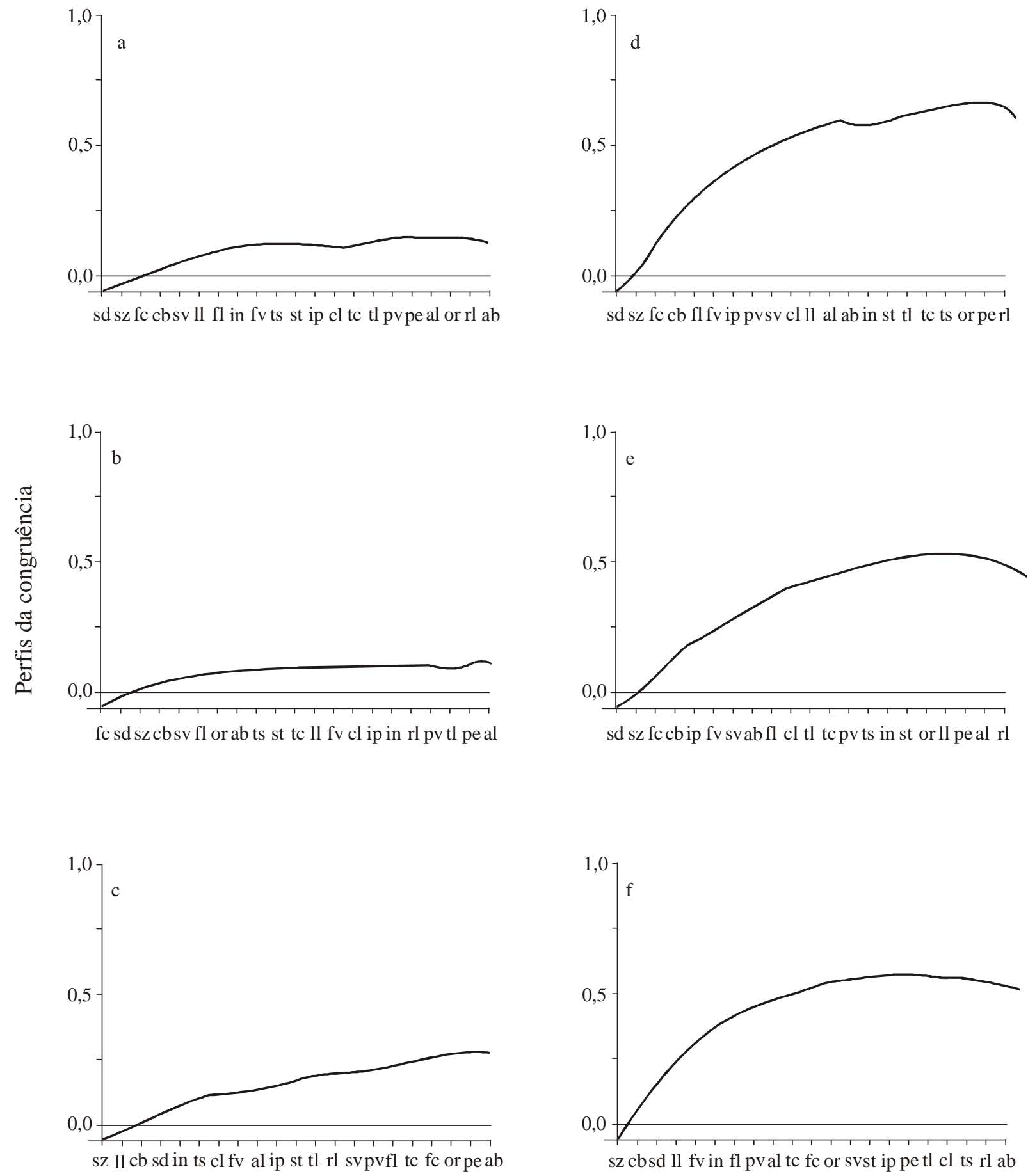

Atributos dos quadros individualizados

Atributos dos quadros agrupados

Figura 1. Perfis da congruência máxima entre a variação da vegetação e a variação de níveis de oferta de forragem, obtidos com os quadros individualizados ( $a, b, c)$ e com os quadros agrupados (d, e, f) dos 21 atributos cumulativos da direita para a esquerda (pg: posição das gemas; pe: persistência; or: órgãos de reserva; sz: sazonalidade; fc: forma de crescimento; ip: inclinação do caule; pv: propagação vegetativa; ab: altura da biomassa; cb: comprimento da bainha; st: secção transversal da lâmina; cl: comprimento da lâmina; ll: largura da lâmina; al: área da lâmina; fl: formato da lâmina; tc: tipo de tecido dos caules aéreos; tl: textura da lâmina; sd: superfície dorsal da lâmina; sv: superfície ventral da lâmina; in: indumento da lâmina; rl: resistência da lâmina à tensão; ts: tensão da lâmina). 
vembro, ou quando se consideram os dois levantamentos conjuntamente. Por esse motivo, e com a intenção de sintetizar as informações nas análises posteriores, optou-se por considerar o levantamento do conjunto completo dos dados (duas épocas conjuntamente) como suficiente.

Desta forma, o nível de agregação e época de amostragem que maximizaram o valor de congruência para o fator oferta de forragem foi o da Figura 1d, com o valor de congruência 0,66 , sendo que resistência da lâmina (rl) e persistência (pe) definiram o subconjunto ótimo de atributos. Cada tipo foi formado pelas populações de planta idênticas quanto aos atributos do subconjunto ótimo (Pillar, 1999). Cada combinação de estados assumidos pelos atributos definiu formas únicas de planta, e podem ser considerados TF porque foram definidos com base em atributos que maximizaram a congruência entre a variação da vegetação e o fator. Cada TF representa a resposta de populações de várias espécies ou de uma só espécie, filogeneticamente relacionadas ou não, ao fator considerado (Tabela 2).

Quando as mesmas análises que maximizaram o valor de congruência para oferta de forragem foram aplicadas com a vegetação descrita pela composição de espécies, os resultados determinaram valores de congruência menores do que os obtidos pela descrição baseada em atributos. Os valores máximos de congruência encontrados para as espécies foram de 0,27 , considerando-se os quadros agregados, e 0,063, considerando os quadros individualmente. Esses valores indicam que não foi encontrada relação entre a variação da composição de espécies das parcelas e o nível de oferta de forragem; valores de congruência negativos entre a descrição baseada nas espécies e os fatores devem ser interpretados como uma ausência de correlação (Pillar 1999).

Em trabalhos similares, foram encontrados alguns dos atributos semelhantes ao deste estudo para avaliar o efeito da intensidade de pastejo. Assim, Boggiano (1995) avaliou a descrição da vegetação por 14 atributos morfológicos e observou que o conjunto que maximizou a congruência (largura da lâmina, tração da lâmina, secção transversal da lâmina e textura da lâmina) apresentou em comum a resistência da lâmina à tração. No trabalho de Quadros (1999), o atributo altura da planta foi o que maximizou a congruência entre vegetação e pastejo. As diferenças encontradas entre os trabalhos podem ser atribuídas à escolha e definição dos atributos utilizados e às diferenças nos níveis dos fatores expe- rimentais. Neste estudo, como se trabalhou com cinco níveis de oferta de forragem, as diferenças entre os níveis não foram tão drásticas como nos estudos anteriores. Isso provavelmente determinou que os subconjuntos ótimos de atributos fossem diferentes de quando se estuda, por exemplo, a presença e ausência de pastejo. Portanto, é importante desenvolver um esquema geral para comparação de estudos baseados em atributos, concordando com uma das conclusões do GCTE (Global Change and Terrestrial Ecosystem task 2.2.2) (Weiher et al., 1999). Contudo, apesar da dificuldade de comparação dos estudos citados para o Rio Grande do Sul, a descrição da vegetação baseada nas espécies apresentou menor diferenciação entre os tratamentos do que a descrição baseada nos atributos.

A ordenação dos potreiros com a composição de TF explicou, nos dois primeiros eixos, $93,8 \%$ da variação total nos dados. Desse porcentual, o eixo I descreveu $88,9 \%$ da variação e o eixo II, $4,9 \%$, demonstrando que a ordenação captou, nos dois primeiros eixos, uma razoável porção de variação total dos dados (Figura 2). Em função disso, na interpretação do diagrama de ordenação foram considerados os TF com grau de associação $0,9(|r|>0,9)$ com os eixos.

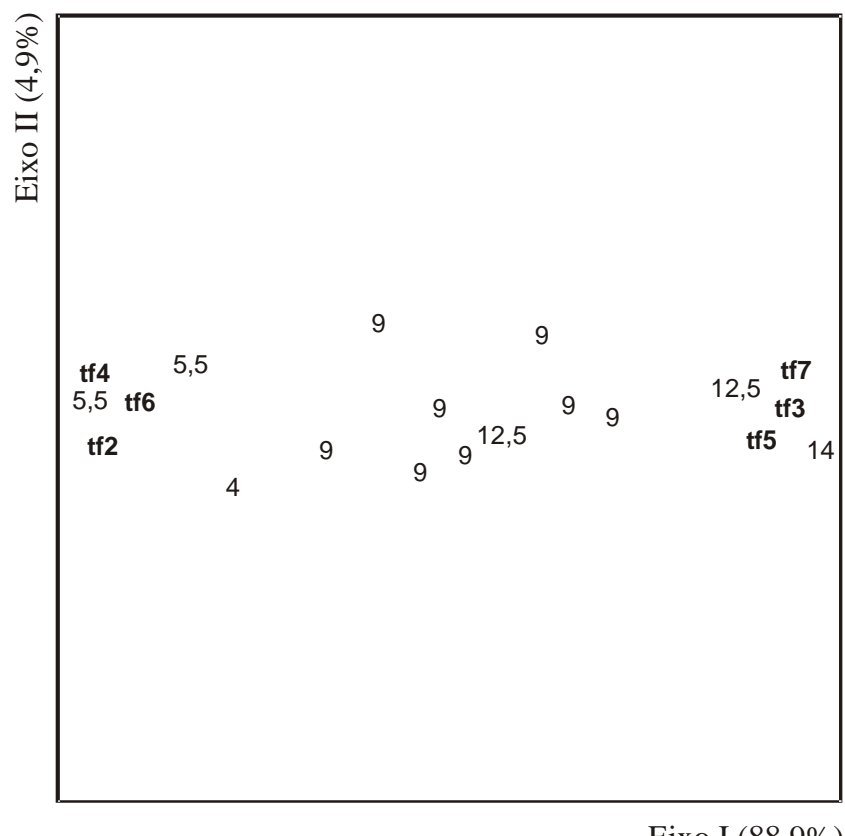

Figura 2. Diagrama de dispersão dos 14 potreiros nos eixos I e II obtidos por análise de coordenadas principais. Os números identificam o nível de oferta de forragem nos potreiros, os quais foram descritos pela composição de oito tipos funcionais. 
No diagrama de ordenação, verifica-se que os potreiros com nível médio do fator $(9,0 \% \mathrm{PV})$ encontram-se dispersos, porém na porção central do eixo I de ordenação (Figura 2). Alguns potreiros com nível de oferta de forragem mais baixo (4 e 5,5\% PV) estão bem à esquerda no diagrama, enquanto os com níveis mais altos estão mais à direita, indicando uma correlação positiva entre a disponibilidade de forragem e escores ao longo do eixo, evidenciando o efeito da intensidade do pastejo.

Com relação aos TF associados negativamente com o eixo I ( $r<0,9)$, identifica-se, após a ordenação, o TF2, TF4 e TF6, formando o que se chamou de Grupo Ecológico 1 (Tabela 2). Esse grupo foi descrito por tipos de plantas com baixa resistência da lâmina à tração, sendo estas persistentes ou não. Díaz et al. (1992) verificaram situação semelhante ao encontrar um tipo graminóide pequeno, com plantas que toleram o pastejo não apresentando mecanismos evidentes de defesa anti herbívoros. Esse grupo de resposta dominante na vegetação sob pastejo, está associado com os potreiros que apresentam baixos valores de disponibilidade de forragem (Figura 2).

Vários estudos têm demonstrado que formas prostradas, meristemas protegidos, folhas pequenas e alto potencial para rebrote aumentam sob pastejo intenso, observando-se o oposto com o pastejo leve (Díaz et al., 1992; Landsberg et al., 1999; McIntyre et al., 1999a). Segundo Coughenour (1985), as gramíneas são adaptadas ao pastejo como resultado de um processo coevolutivo, sendo que a posição dos meristemas basais, a pequena estatura, a grande densidade de perfilhos, a alta taxa de reposição de folhas, a presença de órgãos de reservas subterrâneos e o rápido crescimento permitem a elas evitar ou tolerar tanto as condições de semiaridez como de pastejo.

Em oposição ao Grupo Ecológico 1, os TF3, TF5 e TF7 estão associados positivamente $(r>0,9)$ com o eixo I, formando, após a ordenação, o que se denominou Grupo Ecológico 2 (Tabela 2). Este se caracteriza por tipos de plantas com maior resistência de suas lâminas foliares e persistência, o que pode estar relacionado a um tipo de planta maior, com aumento, principalmente, da lâmina foliar. Esse grupo sintetiza a influência do aumento da oferta de forragem e é dominante em vegetação sob pastejo leve, como nos potreiros que apresentam altos valores de disponibilidade de forragem (Figura 2).

Com esse teste de aleatorização, avaliou-se a hipótese nula de diferença entre os tratamentos, quando se descreveu a vegetação pelos TF. A hipótese nula foi rejeitada $(\mathrm{P}=0,002)$, evidenciando-se, assim, que a descrição da vegetação baseada nos TF possibilita a detecção de diferenças significativas na estrutura da vegetação. Os contrastes significativos entre os níveis de oferta de forragem foram: $4,0 \%$ e $12,5 \% \mathrm{PV}$ $(\mathrm{P}=0,002)$ e $5,5 \%$ e $12,5 \%$ PV $(\mathrm{P}=0,019)$ Os contrastes que apresentaram diferenças significativas com maior probabilidade de erro tipo I foram entre os níveis $4,0 \%$ e $9,0 \%$ PV $(\mathrm{P}=0,034)$ e $4,0 \%$ e $14,0 \%$ $\mathrm{PV}(\mathrm{P}=0,078)$.

\section{Conclusão}

A utilização de tipos funcionais de planta, na descrição da composição das comunidades, pode detectar efeito da intensidade de pastejo (oferta de forragem); esse efeito não é evidenciado na análise das mesmas comunidades baseada na composição de espécies.

\section{Agradecimentos}

Aos professores Gerzy Maraschin e Paulo Carvalho, do Departamento de Plantas Forrageiras e Agrometeorologia, UFRGS, pela disponibilização da área experimental; às professoras Ilsi Boldrini e Sílvia Miotto, do Departamento de Botânica, UFRGS, pela colaboração na identificação das espécies coletadas; à Capes, pela concessão de bolsa de estudos ao primeiro autor; ao CNPq, pela concessão de bolsa de pesquisa ao segundo autor e apoio ao projeto.

\section{Referências}

BOGGIANO, P.R. Dinâmica da produção primária da pastagem nativa em área de fertilidade corrigida sob efeito de adubação nitrogenada e oferta de forragem. 2000. 191p. Tese (Doutorado) - Universidade Federal do Rio Grande do Sul, Porto Alegre.

BOGGIANO, P.R. Relações entre estrutura da vegetação e pastejo seletivo de bovinos em campo natural. 1995. 159p. Dissertação (Mestrado) - Universidade Federal do Rio Grande do Sul, Porto Alegre.

BOX, E.O. Plant functional types and climate at the global scale. Journal of Vegetation Science, v.7, p.309-320, 1996.

BRAUN-BLANQUET. Fitosociología: bases para el estudio de las comunidades vegetales. 3.ed. rev. aum. Madrid: Blume, 1964. 819p.

BRISKE, D.D.; RICHARDS, J.H. Plant responses to defoliation: a physiological, morphological and demographic evaluation. In: BEDUNAH, D.J.; SOSEBEE, R.E. (Ed.). Wildland plants: physiological geology and developmental morphology. Denver: Society for Range Management, 1995. p.635-710. 
BURKART, A. Flora ilustrada de Entre Rios (Argentina). Buenos Aires: Instituto Nacional de Tecnología Agropecuaria, 1969. tomo 6, pt. 2/6. (Colección Científica).

COUGHENOUR, M.B. Graminoid responses to grazing by large herbivores: adaptations, ex-adaptations and interacting processes. Annals of the Missouri Botanical Garden, v.72, p.852-863, 1985.

DÍAZ BARRADAS, M.C.; ZUNZUNEGUI, M.; TIRADO, R.; AIN-LHOUT, F.; GARCÍA NOVO, F. Plant functional types and ecosystem function in a Mediterranean shrubland. Journal of Vegetation Science, v.10, p.709-716, 1999.

DÍAZ, S.; ACOSTA, A.; CABIDO, M. Morphological analysis of herbaceous communities under different grazing regimes. Journal of Vegetation Science, v.3, p.689-696, 1992.

DÍAZ, S.; CABIDO, M.; ZAK, M.; MARTÍNEZ CARRETERO, E.; ARANÍBAR, J. Plant functional traits, ecosystem structure and land-use history along a climatic gradient in central-western Argentina. Journal of Vegetation Science, v.10, p.651-660, 1999.

EMBRAPA. Centro Nacional de Pesquisa de Solos (Rio de Janeiro, RJ). Sistema brasileiro de classificação dos solos. Brasília: Embrapa-SPI; Embrapa-CNPS, 1999. 412p.

GRIME, J.P.; THOMPSON, K.; HUNT, R.; HODGSON, J.G.; CORNELISSEN, J.H.C.; RORISON, I.H.; HENDRY G.A.F.; ASHENDEN, T.W.; ASKEW, A.P.; BAND, S.R.; BOOTH, R.E.; BOSSARD, C.C.; CAMPBELL, B.D.; COOPER, J.E.L.; DAVISON, A.W.; GUPTA, P.L.; HALL, W.; HAND, D.W.; HANNAH, M.A.; HILLIER, S.H.; HODKINSON, D.J.; JALILI, A.; LIU, Z.; MacKEY, J.M.L.; MATTHEWS, N.; MOWFORTH, M.A.; NEAL, A.M.; READER, R.J.; REILING, K.; ROSS-FRASER, W.; SPENCER, R.E.; SUTTON, F.; TASKER, D.E.; THORPE, P.C.; WHITEHOUSE, J. Integrated screening validates primary axes of specialization in plants. Oikos, v.79, p.259-281, 1997.

KEDDY, P.A. Assembly and response rules: two goals for predictive community ecology. Journal of Vegetation Science, v.3, p.157164, 1992.

LANDSBERG, J.; LAVOREL, S.; STOL, J. Grazing response groups among understorey plants in arid rangelands. Journal of Vegetation Science, v.10, p.683-696, 1999.

MAAREL, E. van der. Transformation of cover-abundance values in phytosociology and its effects on community similarity. Vegetatio, v.39, p.97-114, 1979.
McINTYRE, S.; DÍAZ, S.; LAVOREL, S.; CRAMER, W. Plant functional types and disturbance dynamics: introduction. Journal of Vegetation Science, v.10, p.604-608, 1999a.

McINTYRE, S.; LAVOREL, S.; LANDSBERG, J.; FORBES, T.D.A. Disturbance response in vegetation: towards a global perspective on functional traits. Journal of Vegetation Science, v.10, p.621-630, 1999b.

MANTEL, N. The detection of disease clustering and a generalized regression approach. Cancer Research, v.27, p.209-220, 1967.

PILLAR, V.D. On the identification of optimal plant functional types. Journal of Vegetation Science, v.10, p.631-640, 1999.

PILLAR, V.D. SYNCSA: software for character-based community analysis. Porto Alegre: UFRGS, 2000.

PILLAR, V.D.; ORLÓCI, L. Character-based community analysis: the theory and an application program. The Hague: SPB Academic, 1993. 270p. (Ecological Computations Series, 5).

PILLAR, V.D.; ORLÓCI, L. Fuzzy component in community level comparisons. In: FEOLI, E.; ORLÓCI, L. (Ed.). Computer assisted vegetation analysis. Dordrecht: Kluwer, 1991. p.41-46.

PILLAR, V.D.; ORLÓCI, L. On randomization testing in vegetation science: multifactor comparisons of relevé groups. Journal of Vegetation Science, v.7, p.585-592, 1996.

QUADROS, F.L.F. de. Dinâmica vegetacional em pastagem natural submetida a tratamentos de queima e pastejo. 1999. 141p. Tese (Doutorado) - Universidade Federal do Rio Grande do Sul, Porto Alegre.

SKARPE, C. Plant functional types and climatic in a southern African savanna. Journal of Vegetation Science, v.7, p.397-404, 1996.

STEFFEN, W.L.; WALKER, B.H.; INGRAM, J.S.I.; KOCH, G.W. Global change and terrestrial ecosystems: the operational plan: the operational plan. Stockholm: International Geosphere-Biosphere Programme, 1992. 95p. (IGBP Report, 21).

THOMPSON, K.; HILLIER, S.H.; GRIME, J.P.; BOSSARD, C.C.; BAND, S.R. A functional analysis of a limestone grassland community. Journal of Vegetation Science, v.7, p.371-380, 1996.

WEIHER, E.; WERF, A. van der.; THOMPSON, K.; RODERICK, M.; GARNIER, E.; ERIKSSON, O. Challenging theophrastus: a common core list of plant traits for functional ecology. Journal of Vegetation Science, v.10, p.609-620, 1999.

Recebido em 12 de março de 2003 e aprovado em 19 de novembro de 2003 